\title{
On the Existence of Pure-Strategy Equilibria in Large Games*
}

\author{
Guilherme Carmona ${ }^{\dagger}$ \\ Universidade Nova de Lisboa
}

\author{
Konrad Podczeck \\ Universität Wien
}

March 7, 2008

\begin{abstract}
Over the years, several formalizations and existence results for games with a continuum of players have been given. These include those of Schmeidler (1973), Rashid (1983), Mas-Colell (1984), Khan and Sun (1999) and Podczeck (2007a). The level of generality of each of these existence results is typically regarded as a criterion to evaluate how appropriate is the corresponding formalization of large games.

In contrast, we argue that such evaluation is pointless. In fact, we show that, in a precise sense, all the above existence results are equivalent. Thus, all of them are equally strong and therefore cannot rank the different formalizations of large games.
\end{abstract}

Keywords: Nash Equilibrium; Pure Strategies; Approximation; Equilibrium distributions.

*We wish to thank Erik Balder, José Fajardo, Mário Páscoa, Yeneng Sun and Myrna Wooders for very helpful comments and John Huffstot for editorial assistance. Any remaining errors are, of course, ours.

${ }^{\dagger}$ Address: Universidade Nova de Lisboa, Faculdade de Economia, Campus de Campolide, 1099032 Lisboa, Portugal. Email: gcarmona@fe.unl.pt.

${ }^{\ddagger}$ Address: Institut für Wirtschaftswisenschaften, Universität Wien, Hohenstaufengrasse 9, A-1010 Wien, Austria. Email: konrad.podczeck@univie.ac.at 


\section{Introduction}

Nash's (1950) celebrated existence theorem asserts that every finite normal-form game has a mixed strategy equilibrium. However, in many contexts mixed strategies are unappealing and hard to interpret, leading naturally to the question of the existence of pure strategy Nash equilibria.

Schmeidler (1973) was successful in obtaining an answer to the above question. He showed that in a special class of games - in which each player's payoff depends only on his choice and on the average choice of the others - a pure strategy Nash equilibrium exists in every such game with a continuum of players.

Schmeidler's formalization parallels that of Nash in that players have a finite action space, there is a function assigning to each of them a payoff function in a measurable way and the equilibrium notion is formalized in terms of a strategy, i.e., as a measurable function from players into actions. The difference is that, while in Nash (1950) there is a finite number of players (which, in particular, makes the measurability conditions trivial), in Schmeidler (1973) the set of players is the unit interval endowed with the Lebesgue measure.

Although natural, Schmeidler's formalization entails serious difficulties. As shown by Khan, Rath, and Sun (1997), Schmeidler's theorem does not extend to general games - in fact, one has to assume that either the action space or the family of payoff functions is denumerable in order to guarantee the existence of a pure strategy equilibrium (see Khan and Sun (1995b) and Carmona (2008)).

Motivated by this, several alternative formalizations have been proposed in order to obtain an existence result for the case of a general, not necessarily countable, action space. These include those of Khan and Sun (1999) and Podczeck (2007a), which consider a richer measure space of players, that of Mas-Colell (1984), where the equilibrium notion is formalized as a distribution, and that of Rashid (1983), which considers approximate equilibria in games with a large but finite set of players. ${ }^{1}$

\footnotetext{
${ }^{1}$ See also Khan and Sun (1999), Kalai (2004) and Wooders, Cartwright, and Selten (2006) among others.
} 
Clearly, an existence theorem that allows for general compact action spaces also allows for finite such spaces. Furthermore, the existence of an equilibrium strategy also implies the existence of an equilibrium distribution, such distribution being obtained as the inverse image of the equilibrium strategy together with the function assigning payoff functions to players. Therefore, one might be tempted to use the success of a particular formalization to address the existence problem to argue for it as a more appropriate approach to the modeling of large games. We argue that such an appraisal of the different formalizations is misleading by establishing the equivalence between the existence results they yield.

Indeed, our results roughly show that: (1) the existence of approximate equilibria in large games is equivalent to the existence of an equilibrium distribution in games with a continuum of players; (2) the existence of an equilibrium distribution in games with a continuum of players is equivalent to the existence of an equilibrium strategy in games with a super-atomless space of players; and (3) the existence of an equilibrium strategy in games with a super-atomless space of players is equivalent to the existence of an equilibrium strategy in games with a Lebesgue space of players and a finite action space.

The first equivalence result is important because it confirms the fact that games with a continuum of players are an idealization of games with a large but finite set of players. ${ }^{2}$ In fact, as its proof makes clear, equilibria in one class can be constructed using equilibria of the other. The second equivalence result shows formally that, for the solution to the existence problem in games with a super-atomless space of players (which includes those with a Loeb space of players), it makes no difference whether the equilibrium notion is formalized as a strategy or as a distribution. In fact, in such games, a Nash equilibrium exists if and only if an equilibrium distribution exists. Finally, the third equivalence result shows that super-atomless spaces of players provide a rich enough space to solve the (measurability) difficulties that one encounters when working with simpler spaces of players such as Lebesgue spaces.

\footnotetext{
${ }^{2}$ See Carmona (2004a) for a characterization of Nash equilibria of games with continuum of players in terms of approximate equilibria of games with a large but finite set of players.
} 
More broadly, our equivalence results indicate that the assumptions and conclusions present in the several existence theorems for large games compensate each other. Thus, although some of these theorems allow higher levels of generality along some dimensions, this extra generality is exactly compensated by the strengthening of some other condition or the weakening of some other conclusion, rendering all of them as equivalent.

Furthermore, our results provide a non-trivial and unified approach to the existence problem of large games. In fact, they are designed to meet the following two criteria. First, the conditions that we show to be equivalent are stated in such a way that they can be falsified. This goal is obtained by requiring the action space to be merely a separable metric space, rather than compact. Second, by particularizing the action space to be compact, we obtain as a corollary to our results the classical existence theorems of Schmeidler (1973), Mas-Colell (1984), Khan and Sun (1995b) and Khan and Sun (1999).

We note that an equivalence result similar to ours has been obtained by Balder (2002). There, he shows that the existence of equilibrium in pure strategy is equivalent to the existence of equilibrium in mixed strategies (and, like us, uses this result to obtain several known existence results). Our results are different because: (a) the conditions that we show to be equivalence are different than those considered by Balder (2002), (b) his equivalence is between two true propositions, while in ours, the propositions can be true or false, (c) his framework is more general than ours but (d) our arguments are (somewhat) elementary. In contrast with Balder's work, our goal is not to obtain a general existence theorem that can generalize or at least encompass most of such results, but rather to show that several standard formalizations of large games yield equivalent existence results.

The equivalence between the formalizations we consider to yield an existence result is likely to hold more generally. In particular, recent results by Al-Najjar (2007) show that this conclusion can be extended to discrete large games, i.e., games with a countable set of players endowed with a finitely additive distribution.

The paper is organized as follows. In Section 2, we introduce our notation and 
basic definitions. In Section 3, we present our equivalence results. The proof of our main results are presented in Section 4. These proofs rely on three lemmas (stated and proved also in Section 4) that have some interest in their own right. The first provides a characterization of equilibrium distributions in terms of approximate equilibria of games with a large, but finite number of players. The second provides sufficient conditions for the existence of finite-valued approximate equilibria in games with a continuum of players. Finally, the third presents a representation result which implies that, in games with a super-atomless space of players, every equilibrium distribution is the inverse image of a Nash equilibrium and the function describing the game. Section 5 provides some concluding remarks. Some auxiliary results are in the Appendix.

\section{Notation and Definitions}

In the class of normal-form games we consider, all players have a common pure strategy space and each player's payoff depends on his choice and on the distribution of actions induced by the choices of all players. Let $X$ denote the common action space; we assume that $X$ is a separable metric space and let $d$ denote the metric on $X$. A distribution of actions is simply a Borel probability measure on $X$. We let $\mathcal{M}(X)$ denote the set of Borel probability measures on $X$ endowed with the Prohorov metric $\rho$, and let $\mathcal{C}$ denote the space of all bounded, continuous, real-valued functions on $X \times \mathcal{M}(X)$ endowed with the sup norm. ${ }^{3}$ Thus, each player's payoff function is an element of $\mathcal{C}$.

The space of players is described by a probability space $(T, \Sigma, \varphi)$. A game is then specified by the vector of payoff functions, one for each player. To each player $t$, we associate, in a measurable way, a bounded, continuous function $V(t): X \times \mathcal{M}(X) \rightarrow \mathbb{R}$ with the following interpretation: $V(t)(x, \pi)$ is player $t$ 's payoff when he plays action $x$ and faces the distribution $\pi$. Thus, we have defined a function $V: T \rightarrow \mathcal{C}$ and we assume that $V$ is measurable and that it induces a tight probability measure on $\mathcal{C}$. Formally, letting $\mathcal{T}(\mathcal{C})$ denote the set of all tight Borel probability measures on $\mathcal{C}$, we

\footnotetext{
${ }^{3}$ Recall that the Prohorov metric metricizes the weak topology of $\mathcal{M}(X)$.
} 
require that $\varphi \circ V^{-1} \in \mathcal{T}(\mathcal{C})$. In such a game, a strategy is a measurable function $f: T \rightarrow X$. Then, for any strategy $f$, player $t$ 's payoff function is obtained from $V$ in the following way:

$$
U(t)(f)=V(t)\left(f(t), \varphi \circ f^{-1}\right) .
$$

We denote such a game by $G=((T, \Sigma, \varphi), V, X)$.

The following particular cases for the space of players play a special role in our results. Our asymptotic results concern games with a large, but finite set of players. In that case, we denote the space of players by $\left(T_{n}, \Sigma_{n}, \nu_{n}\right)$, where $n$ is the number of players, $T_{n}=\{1, \ldots, n\}, \Sigma_{n}$ equals the family of all subsets of $T_{n}$ and $\nu_{n}$ is the uniform measure on $T_{n}$, i.e., $\nu_{n}(\{t\})=1 / n$ for all $t \in T_{n}$. A game with a finite number of players is then represented by $G_{n}=\left(\left(T_{n}, \Sigma_{n}, \nu_{n}\right), V_{n}, X\right)$. Note that in this case $V_{n}: T_{n} \rightarrow \mathcal{C}$ is measurable and satisfies $\nu_{n} \circ V_{n}^{-1} \in \mathcal{T}(\mathcal{C})$ in a trivial way.

Our asymptotic result also concerns tight families of games with a finite number of players, which are defined as follows. Let $\Gamma$ be a family of games with a finite number of players and, for every game $\gamma \in \Gamma$, let $V_{\gamma}$ be the function describing it and $n_{\gamma}$ be its number of players. We say that $\Gamma$ is a tight family of games with a finite number of players if the family of Borel probability measures $\left\{\nu_{n_{\gamma}} \circ V_{\gamma}^{-1}\right\}_{\gamma \in \Gamma}$ is tight.

In games with a finite number of players, each player has a small but positive impact on the distributions of actions. This is in contrast with the case of games with a continuum of players. Formally, $G=((T, \Sigma, \varphi), V, X)$ is a game with a continuum of players if $(T, \Sigma, \varphi)$ is an atomless probability space.

An important special case for the space of players is obtained when it equals the unit interval $[0,1]$ endowed with the Lebesgue measure $\lambda$ on its Borel $\sigma$-algebra $\mathcal{B}([0,1])$. Another particular case considered in our results is obtained when the space of players is super-atomless. Formally, $(T, \Sigma, \varphi)$ is super-atomless if for every $E \in \Sigma$ with $\varphi(E)>0$, the subspace of $L^{1}(\varphi)$ consisting of the elements of $L^{1}(\varphi)$ vanishing off $E$ is non-separable. This notion was first introduced by Podczeck (2007b).

Given a game $G=((T, \Sigma, \varphi), V, X)$, a strategy $f, x \in X$, and $t \in T$ such that $\{t\} \in \Sigma$, let $f \backslash_{t} x$ denote the strategy obtained if player $t$ changes his choice from $f(t)$ to $x$. Formally, $f \backslash_{t} x$ denotes the strategy $g$ defined by $g(t)=x$, and $g(\tilde{t})=f(\tilde{t})$, 
for all $\tilde{t} \neq t{ }^{4}$ For all measurable subset $S$ of $X$ and $\varepsilon, \eta \geq 0$, we say that $f$ is an $(\varepsilon, \eta)$ - equilibrium of $G$ relative to $S$ if $f(t) \in \bar{S}$ a.e. $t \in T$ and

$$
\varphi\left(\left\{t \in T: U(t)(f) \geq U(t)\left(f \backslash{ }_{t} x\right)-\varepsilon \text { for all } x \in S\right\}\right) \geq 1-\eta \cdot{ }^{5}
$$

Thus, in an $(\varepsilon, \eta)$ - equilibrium relative to $S$, almost all players play an action in the closure of $S$ and only a small fraction of players can gain more than $\varepsilon$ by deviating from $f$ to an action in $S$. A strategy $f$ is an $\varepsilon$ - equilibrium of $G_{n}$ relative to $S$ if it is an $(\varepsilon, \eta)$ - equilibrium relative to $S$ for $\eta=0$. Furthermore, a strategy $f$ is a Nash equilibrium of $G$ relative to $S$ if it is an $\varepsilon$ - equilibrium of $G$ relative to $S$ for $\varepsilon=0$. A strategy $f$ is a Nash equilibrium of $G$ (resp. $(\varepsilon, \eta)$ - equilibrium of $G$ and $\varepsilon-$ equilibrium of $G$ ) if $f$ is a Nash equilibrium of $G$ (resp. $(\varepsilon, \eta)$ - equilibrium of $G$ and $\varepsilon$ - equilibrium of $G$ ) relative to $X$. We note that in the particular case where $S$ is finite, we have that $f$ is a Nash equilibrium of $G$ relative to $S$ if and only if $f$ is a Nash equilibrium of the game $\tilde{G}=((T, \Sigma, \varphi), \tilde{V}, S)$ with $\tilde{V}$ defined by $\tilde{V}(t)=V(t)_{\mid S \times \mathcal{M}(S)}$ for all $t \in T$.

We also describe a game with a continuum of players by a tight Borel probability measure $\mu$ on $\mathcal{C}$. This description is, in fact, equivalent to the one provided above: given $G=((T, \Sigma, \varphi), V, X)$, we obtain a tight Borel probability measure $\mu=\varphi \circ V^{-1} \in$ $\mathcal{T}(\mathcal{C})$; conversely, every probability measure $\mu \in \mathcal{T}(\mathcal{C})$ can be represented by the distribution induced by a function from the unit interval, endowed with the Lebesgue measure on its Borel $\sigma$-algebra, into $\mathcal{C}$, i.e., there exists a measurable function $V:[0,1] \rightarrow \mathcal{C}$ such that $\mu=\lambda \circ V^{-1}$ (see Kallenberg (1997, Lemma 2.22, page 34)).

Given a Borel probability measure $\tau$ on $\mathcal{C} \times X$, we denote by $\tau_{\mathcal{C}}$ and $\tau_{X}$ the marginal distributions of $\tau$ on $\mathcal{C}$ and $X$ respectively. For all subsets $S$ of $X$, the expression $u(x, \tau) \geq u(S, \tau)$ means $u(x, \tau) \geq u\left(x^{\prime}, \tau\right)$ for all $x^{\prime} \in S$. We denote the

\footnotetext{
${ }^{4}$ Note that $U(t)\left(f \backslash_{t} x\right)=V(t)\left(x, \varphi \circ\left(f \backslash_{t} x\right)^{-1}\right)$ if $G$ is a game with a finite number of players, whereas $U(t)\left(f \backslash_{t} x\right)=V(t)\left(x, \varphi \circ f^{-1}\right)$ when $G$ is a game with a continuum of players.

${ }^{5}$ Note that the set $\left\{t \in T: V(t)\left(f(t), \varphi \circ f^{-1}\right) \geq V(t)\left(x, \varphi \circ f^{-1}\right)-\varepsilon\right.$ for all $\left.x \in S\right\}=$ $(V, f)^{-1}\left(\left\{(u, y) \in \mathcal{C} \times X: u\left(y, \varphi \circ f^{-1}\right) \geq u\left(x, \varphi \circ f^{-1}\right)-\varepsilon\right.\right.$ for all $\left.\left.x \in S\right\}\right)$ is measurable. In fact, $\left\{(u, y) \in \mathcal{C} \times X: u\left(y, \varphi \circ f^{-1}\right) \geq u\left(x, \varphi \circ f^{-1}\right)-\varepsilon\right.$ for all $\left.x \in S\right\}$ is closed and $(V, f)$ is measurable (the latter follows from Fremlin (2003, Proposition 418B, p. 111) since $X$ is separable).
} 
Prohorov metric on $\mathcal{M}(\mathcal{C} \times X)$ by $\hat{\rho}$.

Given a game $\mu \in \mathcal{T}(\mathcal{C})$, a measurable subset $S$ of $X$ and $\varepsilon \geq 0$, a Borel probability measure $\tau$ on $\mathcal{C} \times X$ is an $\varepsilon$ - equilibrium distribution of $\mu$ relative to $S$ if $\tau_{\mathcal{C}}=\mu$, $\operatorname{supp}\left(\tau_{X}\right) \subseteq \bar{S}$ and

$$
\tau\left(\left\{(u, x) \in \mathcal{C} \times X: u\left(x, \tau_{X}\right) \geq u\left(S, \tau_{X}\right)-\varepsilon\right\}\right)=1 .
$$

Roughly, in an $\varepsilon$ - equilibrium distribution relative to $S$ almost all players play an action in the closure of $S$ and cannot gain more than $\varepsilon$ by deviating to another action in $S$. An equilibrium distribution of $\mu$ relative to $S$ is an $\varepsilon$ - equilibrium distribution of $\mu$ relative to $S$ for $\varepsilon=0$. An equilibrium distribution of $\mu$ is an equilibrium distribution of $\mu$ relative to $X$. For all $\varepsilon \geq 0$, a Borel probability measure $\xi$ on $X$ is an $\varepsilon-$ equilibrium distribution over actions of $\mu$ if there exists an $\varepsilon$ - equilibrium distribution $\tau$ of $\mu$ such that $\xi=\tau_{X}$. An $\varepsilon-$ equilibrium distribution of $G=((T, \Sigma, \varphi), V, X)$ relative to $S$ is an $\varepsilon$ - equilibrium distribution of $\varphi \circ V^{-1}$ relative to $S$; the notions

of an equilibrium distribution of $G$ relative to $S$, equilibrium distribution of $G$ and $\varepsilon$ - equilibrium distribution over actions of $G$ are defined analogously.

Let $K$ be a subset of $\mathcal{C}$. We say that $K$ is equicontinuous if for all $\eta>0$ there exists a $\delta>0$ such that $\max \{\rho(\pi, \tau), d(x, y)\}<\delta$ implies $|V(x, \pi)-V(y, \tau)|<\eta$ for all $V \in K$ and for all $x, y \in X$ and $\pi, \tau \in \mathcal{M}(X)$ (see Rudin (1976, p. 156)). In our framework, equicontinuity can be interpreted as placing "a bound on the diversity of payoffs" (see Khan, Rath, and Sun (1997)).

\section{Existence of Pure Equilibria in Large Games}

In this section we state our equivalence results. Our first result states that the existence of an equilibrium distribution in games with a continuum of players is equivalent to the existence of approximate equilibria in sufficiently large games.

Theorem 1 Let $X$ be a separable metric space, $M$ be a compact subset of $X$ and $\mathcal{U} \subseteq \mathcal{C}$. Then, the following conditions are equivalent: 
1. For all games with a continuum of players $\mu \in \mathcal{T}(\mathcal{U})$, there exists an equilibrium distribution $\tau$ of $\mu$ such that $\operatorname{supp}\left(\tau_{X}\right) \subseteq M$.

2. For all equicontinuous subsets $K$ of $\mathcal{U}$ and $\varepsilon>0$, there exists $m, N \in \mathbb{N}$ and $\left\{x_{1}, \ldots, x_{m}\right\} \subseteq M$ such that for all $n \geq N$, all games with a finite number of players $G_{n}=\left(\left(T_{n}, \Sigma_{n}, \nu_{n}\right), V_{n}, X\right)$ with $V_{n}\left(T_{n}\right) \subseteq K$ have an $\varepsilon$ - equilibrium $f_{n}$ satisfying $f_{n}\left(T_{n}\right) \subseteq\left\{x_{1}, \ldots, x_{m}\right\}$.

3. For all tight families of games with a finite number of players $\Gamma$ satisfying $\left\{\nu_{n_{\gamma}} \circ\right.$ $\left.V_{\gamma}^{-1}\right\}_{\gamma \in \Gamma} \subseteq \mathcal{T}(\mathcal{U})$ and $\varepsilon, \eta>0$, there exists $m, N \in \mathbb{N}$ and $\left\{x_{1}, \ldots, x_{m}\right\} \subseteq M$ such that for all $n \geq N$, all games $G_{n} \in \Gamma$ have an $(\varepsilon, \eta)$ - equilibrium $f_{n}$ satisfying $f_{n}\left(T_{n}\right) \subseteq\left\{x_{1}, \ldots, x_{m}\right\}$.

This result clearly stresses the relationship between equilibrium distributions of games with a continuum of players and approximate equilibria of large finite games. In fact, Theorem 1 shows that the existence problem for large games can be equivalently addressed either in its exact version in games with a continuum of players or in an approximate version in large (equicontinuous or tight) games.

We remark that the conditions in Theorem 1 are neither always true nor always false. For instance, when $\mathcal{U}=\mathcal{C}$, they hold if and only if $X$ is compact. ${ }^{6}$ Furthermore, they hold if, for example, $\mathcal{U}$ is the subspace of $\mathcal{C}$ consisting of the constant functions.

We note that the compact support assumption used in conditions $1-3$ plays an important role in Theorem 1 since it allows us to obtain equilibrium distributions using a limit argument and to establish the existence of a finite-valued Nash equilibrium in games with a continuum of players. Its existence cannot be dispensed with. In fact, if conditions 1 and 2 were to be changed to

(a) For all games with a continuum of players $\mu \in \mathcal{T}(\mathcal{U})$, there exists an equilibrium distribution $\tau$ of $\mu$

and

\footnotetext{
${ }^{6}$ See the working paper version of this paper for details.
} 
(b) For all equicontinuous subsets $K$ of $\mathcal{U}$ and $\varepsilon>0$, there exists $m, N \in \mathbb{N}$ and $\left\{x_{1}, \ldots, x_{m}\right\} \subseteq X$ such that for all $n \geq N$, all games with a finite number of players $G_{n}=\left(\left(T_{n}, \Sigma_{n}, \nu_{n}\right), V_{n}, X\right)$ with $V_{n}\left(T_{n}\right) \subseteq K$ have an $\varepsilon$ - equilibrium $f_{n}$ satisfying $f_{n}\left(T_{n}\right) \subseteq\left\{x_{1}, \ldots, x_{m}\right\}$,

respectively, then neither would condition (a) imply condition (b) nor would condition (b) imply condition (a) (a similar conclusion holds regarding an analogous variation of condition 3). Thus, Theorem 1 would be false without the compact support requirement.

The above claim is established by the following examples. The first shows that condition (b) does not imply condition (a). Let $X=(0,1), v \in \mathcal{C}$ defined by $v(x, \pi)=$ $x$ for all $x \in X$ and $\pi \in \mathcal{M}(X)$ and let $\mathcal{U}=\{v\}$. Then, for all equicontinuous subsets $K$ of $\mathcal{U}$ and $\varepsilon>0$, let $N=2, m=1$ and $x_{1}=1-\varepsilon$. Thus, $f_{n} \equiv x_{1}$ is an $\varepsilon-$ equilibrium of every game with a finite number of players $G_{n}$ satisfying $V_{n}\left(T_{n}\right) \subseteq \mathcal{U}$. However, it is clear that no $\mu \in \mathcal{T}(\mathcal{U})$ has an equilibrium distribution.

The second example shows that condition (a) does not imply condition (b). Let $X=\mathbb{R}$ with metric $d(x, y)=|x-y| /(1+|x-y|), v_{x} \in \mathcal{C}$ be defined by $v_{x}\left(x^{\prime}, \pi\right)=$ $-d\left(x, x^{\prime}\right)$ for all $x, x^{\prime} \in X$ and $\mathcal{U}=\left\{v_{x}\right\}_{x \in X}$. Let $\mu \in \mathcal{T}(\mathcal{U})$ and $V:[0,1] \rightarrow \mathcal{U}$ be such that $\lambda \circ V^{-1}=\mu$. Note that $h: \mathbb{R} \rightarrow \mathcal{U}$ defined by $h(x)=v_{x}$ is a homeomorphism between $\mathbb{R}$ and $\mathcal{U}$. Then, $f:[0,1] \rightarrow \mathbb{R}$ defined by $f(t)=h^{-1} \circ V$ is a Nash equilibrium of $G=(([0,1], \mathcal{B}([0,1]), \lambda), V, X)$ and so $\tau=\lambda \circ(V, f)^{-1}$ is an equilibrium distribution of $\mu$. Let $K=\mathcal{U}$ and so $K$ is equicontinuous. Since $X$ is not totally bounded, there exists $\varepsilon>0$ such that for all finite subsets $F$ of $X$, there exists $x \in X$ such that $d\left(x, x^{\prime}\right)>\varepsilon$ for all $x^{\prime} \in F$. Let $m, n \in \mathbb{N}$ and $\left\{x_{1}, \ldots, x_{m}\right\} \subseteq X$ be given and let $x \in X$ be such that $d\left(x, x^{\prime}\right)>\varepsilon$ for all $x^{\prime} \in\left\{x_{1}, \ldots, x_{m}\right\}$. Then, letting $n=N$ and $G_{n}$ be such that $V_{n}(t)=v_{x}$ for all $t \in T_{n}$, it follows that if $f_{n}$ is an $\varepsilon$ - equilibrium of $G_{n}$, then $f_{n}(t) \notin\left\{x_{1}, \ldots, x_{m}\right\}$.

Our second equivalence result states that the existence of an equilibrium distribution in games with a continuum of players is equivalent to the existence of a Nash equilibrium in games with a super-atomless space of players. 
Theorem 2 Let $X$ be a separable metric space and $\mathcal{U} \subseteq \mathcal{C}$. Then, the following conditions are equivalent:

1. An equilibrium distribution exists for all games with a continuum of players $\mu \in \mathcal{T}(\mathcal{U})$.

2. A Nash equilibrium exists for all games $G=((T, \Sigma, \varphi), V, X)$ with $V(T) \subseteq \mathcal{U}$ and a super-atomless probability space of players.

Theorem 2 implies that the existence of pure strategy Nash equilibria in games with a super-atomless space of players can be addressed either in terms of strategies or in terms of distributions. Furthermore, it shows that super-atomless spaces are rich enough to solve the measurability problems that one encounters when working with simpler spaces, and which prevent, in general, the existence of an equilibrium strategy.

Our third equivalence result states that the existence of an equilibrium distribution in games with a continuum of players is equivalent to the existence of a Nash equilibrium in games with a finite action space and a Lebesgue space of players. Such an equivalence is obtained through the use of relative equilibrium since it allows for the common action space $X$ to be used in both statements even though $X$ may not be finite. Ideally, the statement would assert the equivalence of the following two conditions:

For all games with a continuum of players $\mu \in \mathcal{T}(\mathcal{U})$ and all closed subsets $S$ of $X$, there exists an equilibrium distribution $\tau$ of $\mu$ relative to $S$,

and

For all games with a continuum of players $G=(([0,1], \mathcal{B}([0,1]), \lambda), V, X)$ with $V([0,1]) \subseteq \mathcal{U}$ and all finite subsets $F$ of $X$, there exists a Nash equilibrium $f$ of $G$ relative to $F$.

However, as in Theorem 1 (and as explained below), a common compact support assumption is needed for such result. But assuming the existence of a compact subset 
$M$ of $X$ such that $\operatorname{supp}\left(\tau_{X}\right) \subseteq M$ and $\operatorname{supp}\left(\lambda \circ f^{-1}\right) \subseteq M$ is not enough. In fact, when $X$ is not compact, there is a finite subset $F$ of $X$ such that $F \cap M=\emptyset$, and so neither $\mu$ nor $G$ have an equilibrium relative to $F$; thus, without assuming that both $M \cap S$ and $M \cap F$ are nonempty, the above conditions would be false even when a common compact support is assumed.

Theorem 3 Let $X$ be a separable metric space, $M$ be a compact subset of $X$ and $\mathcal{U} \subseteq \mathcal{C}$. Then, the following conditions are equivalent:

1. For all games with a continuum of players $\mu \in \mathcal{T}(\mathcal{U})$ and all closed subsets $S$ of $X$ such that $M \cap S$ is nonempty, there exists an equilibrium distribution $\tau$ of $\mu$ relative to $S$ such that $\operatorname{supp}\left(\tau_{X}\right) \subseteq M$.

2. For all games with a continuum of players $G=(([0,1], \mathcal{B}([0,1]), \lambda), V, X)$ with $V([0,1]) \subseteq \mathcal{U}$ and all countable, closed subsets $C$ of $X$ such that $M \cap C$ is nonempty, there exists a Nash equilibrium $f$ of $G$ relative to $C$ such that $\lambda \circ$ $f^{-1} \subseteq M$.

3. For all games with a continuum of players $G=(([0,1], \mathcal{B}([0,1]), \lambda), V, X)$ with $V([0,1]) \subseteq \mathcal{U}$ and all finite subsets $F$ of $X$ such that $M \cap F$ is nonempty, there exists a Nash equilibrium $f$ of $G$ relative to $F$ such that $\lambda \circ f^{-1} \subseteq M$.

This result shows that although Lebesgue spaces are restrictive in that they require finite or countable action spaces for the existence of a Nash equilibrium, such a result is strong enough to derive the existence of an equilibrium distribution, and due to Theorem 2, a Nash equilibrium of games with a richer space of players.

As in Theorem 1, the existence of a compact support (i.e., the compact subset of $M$ of $X$ ) cannot be dispensed with in Theorem 3. However, unlike Theorem 1, dropping this requirement from conditions 1 and 2 produces two conditions that are still equivalent and that imply the one resulting from dropping the same requirement from condition 3. Moreover, the example used above to show that condition (b) does not imply condition (a) can still be used to show that the converse is not true. 
Our existence results provide a unifying approach to the existence problem in large games. In fact, by considering the particular case when $X$ is compact and $\mathcal{U}=\mathcal{C}$, we obtain the classical existence results of Schmeidler (1973), Mas-Colell (1984), Khan and Sun (1995b) and Khan and Sun (1999). It is interesting to note that each of these existence theorems can be coupled with our main results to derive the others. Thus, for instance, Schmeidler's theorem, together with Theorem 3, implies the result in Khan and Sun (1995b) (simply by taking $X$ to be countable and $M=C=X$ ) and the one in Mas-Colell (1984) (simply by taking $X$ to be an arbitrarily compact space and $M=S=X$ ). This conclusion, together with Theorem 2, then implies the existence of a Nash equilibrium in games with super-atomless space of players, and so, in particular, in games with an atomless Loeb space of players. Thus, we obtain the existence result for games with a continuum of players in Khan and Sun (1999). Furthermore, by Theorem 1, it also implies their existence result for tight games with a finite but sufficiently large number of players.

Corollary 1 Suppose that $X$ is compact. Then,

1. For all equicontinuous subsets $K$ of $\mathcal{C}$ and $\varepsilon>0$, there exists $m, N \in \mathbb{N}$ and $\left\{x_{1}, \ldots, x_{m}\right\} \subseteq X$ such that for all $n \geq N$, all games with a finite number of players $G_{n}=\left(\left(T_{n}, \Sigma_{n}, \nu_{n}\right), V_{n}, X\right)$ with $V_{n}\left(T_{n}\right) \subseteq K$ have an $\varepsilon$ - equilibrium $f_{n}$ satisfying $f_{n}\left(T_{n}\right) \subseteq\left\{x_{1}, \ldots, x_{m}\right\}$.

2. (Khan and Sun) For all tight families of games with a finite number of players $\Gamma$, and $\varepsilon, \eta>0$, there exists $m, N \in \mathbb{N}$ and $\left\{x_{1}, \ldots, x_{m}\right\} \subseteq X$ such that for all $n \geq N$, all games $G_{n} \in \Gamma$ have an $(\varepsilon, \eta)$ - equilibrium $f_{n}$ satisfying $f_{n}\left(T_{n}\right) \subseteq$ $\left\{x_{1}, \ldots, x_{m}\right\}$.

3. (Mas-Colell) An equilibrium distribution exists for all games with a continuum of players $\mu \in \mathcal{M}(\mathcal{C})$.

4. A Nash equilibrium exists for all games $G=((T, \Sigma, \varphi), V, X)$ with a superatomless probability space of players. 
5. (Khan and Sun) A Nash equilibrium exists for all games $G=((T, \Sigma, \varphi), V, X)$ with an atomless Loeb probability space of players.

6. (Khan and Sun) A Nash equilibrium exists for all games with a continuum of players $G=(([0,1], \mathcal{B}([0,1]), \lambda), V, X)$ with $X$ countable.

7. (Schmeidler) A Nash equilibrium exists for all games with a continuum of players $G=(([0,1], \mathcal{B}([0,1]), \lambda), V, X)$ with $X$ finite.

\section{Proofs and Further Results}

The proof of our main results, Theorems $1-3$, relies on three lemmas which have some interest in their own right. The first provides a characterization of equilibrium distributions in terms of approximate equilibria of games with a large, but finite number of players. Lemma 2 provides sufficient conditions for the existence of finitevalued approximate equilibria in games with a continuum of players. Finally, Lemma 3 presents a representation result which implies that, in games with a super-atomless space of players, every equilibrium distribution is the inverse image of a Nash equilibrium and the function describing the game. These lemmas are stated and proved in Subsections 4.1, 4.2 and 4.3, respectively, while the proofs of Theorems 1, 3 and 2 are presented in Subsections 4.4, 4.5 and 4.6, respectively.

\subsection{A Characterization of Equilibrium Distributions}

In this section we characterize the equilibrium distributions, supported on a given finite set, of some simple games with a continuum of players. These are games with a finite number of characteristics and with payoff functions selected from an equicontinuous family. Despite all these restrictive assumptions, this result is enough to deduce the existence of pure strategy approximate equilibria in large finite games from the existence of an equilibrium distribution with compact support in games with a continuum of players. 
The following notation is used in Lemma 1. When $F$ is a finite set and $\pi$ a probability measure on $F$, we write $\pi_{l}$ instead of $\pi(\{l\})$, whenever $l \in F$, and also $\pi=\left(\pi_{1}, \ldots, \pi_{L}\right)$, with $L=|F|$. This notation also suggests that a measure with a finite support can be thought of as a vector in some Euclidean space. We will also write $\|\pi\|=\max _{l \in F}\left|\pi_{l}\right|$, i.e., $\|\pi\|$ is the sup norm of the vector $\left(\pi_{1}, \ldots, \pi_{L}\right)$. Note that a sequence of measures $\left\{\pi_{n}\right\}_{n=1}^{\infty}$ on $F$ converges to $\pi$ if and only if $\lim _{n \rightarrow \infty}\left\|\pi_{n}-\pi\right\|=0$. Furthermore, for all equicontinuous subsets $K$ of $\mathcal{C}$ and $V \in K$, let $\omega_{V}: \mathbb{R}_{++} \rightarrow \mathbb{R}_{+}$, defined by $\omega_{V}(\delta)=\sup \{|V(x, \pi)-V(y, \tau)|: \max \{d(x, y), \rho(\pi, \tau)\} \leq \delta\}$ for all $\delta>0$, denote the modulus of continuity of $V$ and $\omega_{K}(\delta)=\sup _{V \in K} \omega_{V}(\delta)$. Of course, since $K$ is equicontinuous, then $\lim _{\delta \rightarrow 0} \omega_{K}(\delta)=0$.

Lemma 1 Let $S$ be a finite subset of $X, m=|S|$ and $K$ be an equicontinuous subset of $\mathcal{U}$. Then, the following holds for all games $G=((T, \Sigma, \varphi), V, X)$ with a continuum of players such that $V(T)$ is a finite subset of $K$ and for all $\varepsilon \geq 0$ :

A Borel probability measure $\xi$ on $X$ is an $\varepsilon$ - equilibrium distribution over actions of $G$ with $\operatorname{supp}(\xi) \subseteq S$ if and only if for all games $G_{n}=\left(\left(T_{n}, \Sigma_{n}, \nu_{n}\right), V_{n}, X\right)$ with a finite number of players in which $V_{n}\left(T_{n}\right)$ is a subset of $V(T)$ there exists a strategy $f_{n}: T_{n} \rightarrow S$ such that

1. $f_{n}$ is an $\varepsilon+2 \omega_{K}\left(m\left\|\varphi \circ V^{-1}-\nu_{n} \circ V_{n}^{-1}\right\|+\left(m^{2}+1\right) / n\right)-$ equilibrium of $G_{n}$ and

2. $\left\|\nu_{n} \circ f_{n}^{-1}-\xi\right\| \leq\left\|\varphi \circ V^{-1}-\nu_{n} \circ V_{n}^{-1}\right\|+\frac{m}{n}$.

In order to illustrate the idea of Lemma 1, consider the particular case of a sequence of games $\left\{G_{n}\right\}$ with a finite number of players with $V_{n}\left(T_{n}\right) \subseteq V(T)$ and with $\left\|\varphi \circ V^{-1}-\nu_{n} \circ V_{n}^{-1}\right\|$ converging to zero. In this case, we can, intuitively, say that the sequence $\left\{G_{n}\right\}$ converges to $G$. If $\xi$ is an equilibrium distribution over actions of $G$, then Lemma 1 guarantees the existence of an $\varepsilon_{n}$ - equilibrium $f_{n}$ of $G_{n}$ satisfying $\varepsilon_{n} \rightarrow 0$ and $\left\|\nu_{n} \circ f_{n}^{-1}-\xi\right\| \rightarrow 0$. That is, finite games that are close to $G$ have approximate equilibria, with a degree of approximation close to zero, whose induced distributions are close to $\xi$. 
Conversely, the existence of approximate equilibria of games converging to $G$, with a vanishing degree of approximation and with induced distributions converging to $\xi$, is enough to show that $\xi$ is an equilibrium distribution over actions of $G$.

The strength of Lemma 1, which is crucial to the asymptotic result, is that the degree of approximation involved depends only on $\varepsilon$, on the equicontinuous family $K$, on the number of pure strategies $m$ of the set $S$, on the Euclidean distance between the distributions of characteristics $\left\|\varphi \circ V^{-1}-\nu_{n} \circ V_{n}^{-1}\right\|$ and on the number of players $n$. In particular, it is independent of the particular games $G$ and $G_{n}$ that we are considering. So, if $\varepsilon$ and the set of actions is fixed, and we are considering games $G$ and $G_{n}$ with the same distribution of characteristics, then the degree of approximation depends only on $n$. This fact is at the core of our asymptotic result: once $n$ is sufficiently large, equilibrium distributions of $G$ induce approximate equilibria of $G_{n}$.

Proof of Lemma 1. Let $S=\left\{x_{1}, \ldots, x_{m}\right\}$ be a finite subset of $X$ and $K$ be an equicontinuous subset of $\mathcal{U}$. Let $\varepsilon \geq 0$ and let $G=((T, \Sigma, \varphi), V, X)$ be a game with a continuum of players such that $V(T)$ is a finite subset of $K$. Let $\beta=\varphi \circ V^{-1}$ and let $\operatorname{supp}(\beta)=\left\{V_{1}, \ldots, V_{L}\right\}$.

(Necessity) Let $\xi$ be an $\varepsilon$ - equilibrium distribution over actions of $G$ with $\operatorname{supp}(\xi) \subseteq$ $S$ and let $\psi$ be an $\varepsilon$ - equilibrium distribution of $G$ such that $\xi=\psi_{X}$. For all $1 \leq l \leq L$ and $1 \leq i \leq m$, let $\psi_{l, i}=\psi\left(\left\{\left(V_{l}, x_{i}\right)\right\}\right)$ and note that $\sum_{i=1}^{m} \psi_{l, i}=\beta_{l}$ and $\sum_{l=1}^{L} \psi_{l, i}=\xi_{i}$. Since $\psi$ is an $\varepsilon$ - equilibrium distribution, it follows that if $\psi_{l, i}>0$ then, for all $x \in X$,

$$
V_{l}\left(x_{i}, \xi\right) \geq V_{l}(x, \xi)-\varepsilon
$$

Let $G_{n}$ be a game with a finite number of players such that $V_{n}\left(T_{n}\right)$ is a subset of $V(T)$. For all $1 \leq l \leq L$, let $T_{n, l}=\left\{t \in T_{n}: V_{n}(t)=V_{l}\right\}$ and $\gamma_{n, l}=\left|T_{n, l}\right|$. Then, $\gamma_{n}=\left(\gamma_{n, 1}, \ldots, \gamma_{n, L}\right)$ is such that $\gamma_{n} / n=\nu_{n} \circ V_{n}^{-1}$.

Let $1 \leq l \leq L$ be given. Define $E_{l}=\left\{e_{i} \in E: \psi_{l, i}>0\right\}$, where $E=\left\{e_{1}, \ldots, e_{m}\right\}$ is the standard basis of $\mathbb{R}^{m}$. Define $E_{t}=E_{l}$ if $t \in T_{n, l}$. If $\gamma_{n, l}>0$, it follows that, $E_{l} \subseteq \frac{1}{\gamma_{n, l}} \sum_{t \in T_{n, l}} E_{t}$. Also, we have that $\psi_{l} / \beta_{l}=\left(\psi_{l, 1} / \beta_{l}, \ldots, \psi_{l, m} / \beta_{l}\right) \in \operatorname{co}\left(E_{l}\right)$ and so $\psi_{l} / \beta_{l} \in \operatorname{co}\left(\frac{1}{\gamma_{n, l}} \sum_{t \in T_{n, l}} E_{t}\right)=\frac{1}{\gamma_{n, l}} \sum_{t \in T_{n, l}} \operatorname{co}\left(E_{t}\right)$. 
Define

$$
\tau=\sum_{l=1}^{L} \frac{\gamma_{n, l}}{n} \frac{\psi_{l}}{\beta_{l}}=\sum_{l: \gamma_{n, l}>0} \frac{\gamma_{n, l}}{n} \frac{\psi_{l}}{\beta_{l}}
$$

Then, for all $1 \leq i \leq m$, it follows that $\left|\xi_{i}-\tau_{i}\right| \leq \sum_{l=1}^{L} \frac{\psi_{l, i}}{\beta_{l}}\left|\frac{\gamma_{n, l}}{n}-\beta_{l}\right| \leq\left\|\beta-\frac{\gamma_{n}}{n}\right\|$, and so $\|\xi-\tau\| \leq\left\|\beta-\frac{\gamma_{n}}{n}\right\|$. Hence, by Lemma 4 ,

$$
\rho(\xi, \tau) \leq m\left\|\beta-\frac{\gamma_{n}}{n}\right\|
$$

Furthermore, $\tau \in \sum_{l: \gamma_{n, l}>0} \frac{\gamma_{n, l}}{n} \frac{1}{\gamma_{n, l}} \sum_{t \in T_{n, l}} \operatorname{co}\left(E_{t}\right)=\frac{1}{n} \sum_{t \in T_{n}} \operatorname{co}\left(E_{t}\right)$. Thus, by the Shapley-Folkman Theorem (see Rashid (1983, p. 9)), it follows that there are $n$ points $\left(\alpha_{t}\right)_{t \in T_{n}}$ such that $\alpha_{t} \in \operatorname{co}\left(E_{t}\right)$ for all $t \in T_{n},\left|\left\{t \in T_{n}: \alpha_{t} \notin E_{t}\right\}\right| \leq m$ and

$$
\tau=\frac{1}{n} \sum_{t \in T_{n}} \alpha_{t}
$$

Let $1 \leq l \leq L$ and define $P_{n}=\left\{t \in T_{n}: \alpha_{t} \in E\right\}$. Define a strategy $f_{n}$ as follows: if $t \in P_{n}$, then let $e_{i}$ be such that $\alpha_{t}=e_{i}$ and define $f_{n}(t)=x_{i}$; if $t \in P_{n}^{c}:=T_{n} \backslash P_{n}$ and $V_{t}=V_{l}$, choose $1 \leq i \leq m$ such that $\psi_{l, i}>0$ and define $f_{n}(t)=x_{i}$. By (4), it follows that $V(t)\left(f_{n}(t), \xi\right) \geq V(t)(x, \xi)-\varepsilon$ for all $t \in T_{n}$ and $x \in X$.

Let $\sigma=\nu_{n} \circ f_{n}^{-1}$. We claim that $\|\tau-\sigma\| \leq \frac{m}{n}$. In fact, for all $1 \leq i \leq m$ we have that

$$
\sigma_{i}=\sum_{t \in P_{n}} \frac{\alpha_{t, i}}{n}+\frac{\left|P_{n}^{c} \cap f_{n}^{-1}\left(x_{i}\right)\right|}{n}=\sum_{t \in P_{n}} \frac{\alpha_{t, i}}{n}+\sum_{t \in P_{n}^{c} \cap f_{n}^{-1}\left(x_{i}\right)} \frac{1}{n}
$$

and $\tau_{i}=\sum_{t=1}^{n} \alpha_{t, i} / n$. Therefore, letting $\chi_{f^{-1}\left(x_{i}\right)}$ denote the characteristic function of $f^{-1}\left(x_{i}\right)$, we obtain that $\left|\tau_{i}-\sigma_{i}\right|=\frac{1}{n}\left|\sum_{t \in P_{n}^{c}}\left(\alpha_{t, i}-\chi_{f^{-1}\left(x_{i}\right)}(t)\right)\right| \leq \frac{\left|P_{n}^{c}\right|}{n} \leq \frac{m}{n}$ and so $\|\tau-\sigma\| \leq m / n$

Since $\nu_{n} \circ f^{-1}=\sigma$ and $\|\xi-\tau\| \leq\left\|\beta-\gamma_{n} / n\right\|$, then $\left\|\nu_{n} \circ f^{-1}-\xi\right\| \leq\left\|\beta-\gamma_{n} / n\right\|+m / n$. This establishes assertion 2 in the statement of the Lemma.

By Lemma 4, $\rho\left(\tau, \nu_{n} \circ f_{n}^{-1}\right) \leq m^{2} / n$ since $\nu_{n} \circ f_{n}^{-1}=\sigma$. Also, by Lemma 5 , it follows that $\rho\left(\nu_{n} \circ f_{n}^{-1}, \nu_{n} \circ\left(f_{n} \backslash_{t} x\right)^{-1}\right) \leq 1 / n$ for all $t \in T_{n}$ and $x \in X$. Hence, using (6), it follows that $\rho\left(\nu_{n} \circ f_{n}^{-1}, \xi\right) \leq m\left\|\beta-\gamma_{n} / n\right\|+m^{2} / n$ and

$$
\rho\left(\nu_{n} \circ\left(f_{n} \backslash_{t} x\right)^{-1}, \xi\right) \leq m\left\|\beta-\frac{\gamma_{n}}{n}\right\|+\frac{m^{2}+1}{n} .
$$


For convenience, let $\theta=m\left\|\beta-\frac{\gamma_{n}}{n}\right\|+\frac{m^{2}+1}{n}$. Hence, for all $t \in T_{n}$ and $x \in X$, we obtain

$$
\begin{aligned}
& V(t)\left(f_{n}(t), \nu_{n} \circ f_{n}^{-1}\right) \geq V(t)\left(f_{n}(t), \xi\right)-\omega_{K}(\theta) \\
& \geq V(t)(x, \xi)-\varepsilon-\omega_{K}(\theta) \geq V(t)\left(x, \nu_{n} \circ\left(f_{n} \backslash t x\right)^{-1}\right)-\varepsilon-2 \omega_{K}(\theta) .
\end{aligned}
$$

Therefore, $f_{n}$ is an $\varepsilon+2 \omega_{K}\left(m\left\|\beta-\gamma_{n} / n\right\|+\left(m^{2}+1\right) / n\right)-$ equilibrium of $G_{n}$.

(Sufficiency) Let $\xi$ be a distribution over $X$ satisfying the condition. Let $\left\{q_{n}\right\} \subseteq$ $\mathbb{Q}_{+}^{L}$ be such that $q_{n} \rightarrow \beta$. For all $n \in \mathbb{N}$, there exist $\gamma_{n}=\left(\gamma_{n, 1}, \ldots, \gamma_{n, L}\right) \in \mathbb{N}^{L}$ and $k_{n} \in \mathbb{N}$ such that $q_{n}=\gamma_{n} / k_{n}$. By multiplying both $k_{n}$ and $\gamma_{n}$ by $n$ if necessary, we may assume that $k_{n} \geq n$. Define, for all $n$, a game $G_{k_{n}}=\left(\left(T_{k_{n}}, \Sigma_{k_{n}}, \nu_{k_{n}}\right), V_{k_{n}}, X\right)$ where $V_{k_{n}}$ satisfies $\left|\left\{t \in T_{k_{n}}: V_{k_{n}}(t)=V_{l}\right\}\right|=\gamma_{n, l}$ for all $1 \leq l \leq L$.

For all $n$, let $f_{k_{n}}$ satisfy 1 and 2. Consider the sequence $\left\{\nu_{k_{n}} \circ\left(V_{k_{n}}, f_{k_{n}}\right)^{-1}\right\}_{n} \subseteq$ $\mathcal{M}\left(\left\{V_{1}, \ldots, V_{L}\right\} \times S\right)$. Since $\mathcal{M}\left(\left\{V_{1}, \ldots, V_{L}\right\} \times S\right)$ is compact, taking a subsequence if necessary, we may assume that it converges. Let $\tau=\lim _{n} \nu_{k_{n}} \circ\left(V_{k_{n}}, f_{k_{n}}\right)^{-1}$. Then, $\tau_{\mathcal{U}}=\beta=\lambda \circ V^{-1}, \tau_{X}=\xi$ and $\operatorname{supp}(\xi) \subseteq S$ since, respectively, $\nu_{k_{n}} \circ V_{k_{n}}^{-1}=\gamma_{n} / k_{n} \rightarrow \beta$, $\left\|\nu_{k_{n}} \circ f_{k_{n}}^{-1}-\xi\right\| \leq\left\|\beta-\gamma_{n} / k_{n}\right\|+m / k_{n} \rightarrow 0$ and $f_{k_{n}}\left(T_{k_{n}}\right) \subseteq S$ for all $n$. Since $\nu_{k_{n}} \circ\left(V_{k_{n}}, f_{k_{n}}\right)^{-1}$ converges to $\tau, f_{k_{n}}$ is an $\varepsilon+2 \omega_{K}\left(m\left\|\beta-\frac{\gamma_{n}}{k_{n}}\right\|+\frac{m^{2}+1}{k_{n}}\right)-$ equilibrium of $G_{k_{n}}$ and $\lim _{n}\left(m\left\|\beta-\frac{\gamma_{n}}{k_{n}}\right\|+\frac{m^{2}+1}{k_{n}}\right)=0$, it follows, by Lemma 6 , that $\tau$ is an $\varepsilon-$ equilibrium distribution of $G$. Thus, $\xi$ is an $\varepsilon$ - equilibrium distribution over action of $G$ with $\operatorname{supp}(\xi) \subseteq S$.

\subsection{Finite-valued Equilibria}

In this subsection we address the existence of finite-valued approximate equilibria. Lemma 2 considers games with a continuum of players where the set of players' characteristics is a countable subset of an equicontinuous family. It guarantees the existence of a finite set of actions with the property that all such games have an approximate equilibrium strategy taking values in this finite set. The strength of this result is that the finite set works uniformly for all such games, i.e., it depends only on the equicontinuous set and on the degree of approximation desired. Since Lemma 1 only applies to games with finite action space, these properties are useful in order 
to demonstrate part of Theorem 1 using that lemma.

Lemma 2 Let $M$ be a compact subset of $X$ and $K$ be an equicontinuous subset of $\mathcal{U}$. Then, for all $\varepsilon>0$, there exists a finite subset $\left\{x_{1}, \ldots, x_{m}\right\}$ of $M$ such that the following holds:

If $\tau$ is an equilibrium distribution of a game with a continuum of players $G=$ $((T, \Sigma, \varphi), V, X)$ with $V(T) \subseteq K$ countable and satisfies $\operatorname{supp}\left(\tau_{X}\right) \subseteq M$, then there exists an $\varepsilon-$ equilibrium strategy $g$ such that $g(T) \subseteq\left\{x_{1}, \ldots, x_{m}\right\}$ and $\hat{\rho}\left(\varphi \circ(V, g)^{-1}, \tau\right)<$ $\varepsilon$

Proof. Let $\varepsilon>0$. Since $K$ is equicontinuous, there exists $\delta>0$ such that $\max \{d(x, y), \rho(\pi, \psi)\}<\delta$ implies that $|u(x, \pi)-u(y, \psi)|<\varepsilon / 2$ for all $x, y \in X$, $\pi, \psi \in \mathcal{M}(X)$ and $u \in K$. We can choose $\delta<\varepsilon$.

Let $\left\{x_{1}, \ldots, x_{m}\right\} \subseteq M$ be such that $M \subseteq \cup_{j=1}^{m} B_{\delta / 2}\left(x_{j}\right)$. Define $B_{1}=B_{\delta / 2}\left(x_{1}\right)$ and $B_{j}=B_{\delta / 2}\left(x_{j}\right) \backslash\left(\cup_{l=1}^{j-1} B_{\delta / 2}\left(x_{l}\right)\right)$ for all $2 \leq j \leq m$.

Let $G=((T, \Sigma, \varphi), V, X)$ be a game with a continuum of players such that $V(T)$ is a countable subset of $K$ and let $\tau$ be an equilibrium distribution of $G$. It follows from Carmona (2008, Theorem 1) that there exists a Nash equilibrium $f$ of $G$ such that $\tau=\varphi \circ(V, f)^{-1}$ and $f(T) \subseteq M$.

Define $g: T \rightarrow\left\{x_{1}, \ldots, x_{m}\right\}$ by $g(t)=x_{j}$ if $f(t) \in B_{j}$. Then, $g$ is measurable and $d(f(t), g(t))<\delta / 2$ for all $t \in T$. This implies that $\{t \in T:(V(t), g(t)) \in$ $D\} \subseteq\left\{t \in T:(V(t), f(t)) \in \bar{B}_{\delta / 2}(D)\right\}$ for all Borel measurable subsets $D$ of $\mathcal{U} \times X$ and so $\varphi \circ(V, g)^{-1}(D) \leq \varphi \circ(V, f)^{-1}\left(\bar{B}_{\delta / 2}(D)\right)+\delta / 2$. Similarly, one can show that $\varphi \circ(V, f)^{-1}(D) \leq \varphi \circ(V, g)^{-1}\left(\bar{B}_{\delta / 2}(D)\right)+\delta / 2$. Thus, $\hat{\rho}\left(\varphi \circ(V, g)^{-1}, \tau\right) \leq \delta / 2<\varepsilon$. Analogously, we can show that $\rho\left(\varphi \circ g^{-1}, \varphi \circ f^{-1}\right) \leq \delta / 2<\delta$.

This implies that for almost all $t \in T$ and all $x \in X, V(t)\left(g(t), \varphi \circ g^{-1}\right)>$ $V(t)\left(f(t), \varphi \circ f^{-1}\right)-\varepsilon / 2 \geq V(t)\left(x, \varphi \circ f^{-1}\right)-\varepsilon / 2>V(t)\left(x, \varphi \circ g^{-1}\right)-\varepsilon$. Hence, $g$ is an $\varepsilon$ - equilibrium of $G$. 


\subsection{A Representation Result for Distributions}

In this subsection, we characterize equilibrium distributions of games with a superatomless space of players in terms of its Nash equilibria. Such a characterization (Corollary 2) is a direct consequence of Lemma 3, which is a representation result for tight measures. In general, every tight measure $\tau$ on a metric $Y$ can be represented as the inverse image of a measurable function $h$, mapping the unit interval into $Y$. However, if $Y=A \times B$ and $\tau_{A}=\lambda \circ g^{-1}$, where $g:[0,1] \rightarrow A$, in general, there is no $f:[0,1] \rightarrow B$ such that $\tau=\lambda \circ(g, f)^{-1}$. Lemma 3 shows that the existence of such a function $f$ provided that the probability space $([0,1], \mathcal{B}([0,1]), \lambda)$ is replaced by a super-atomless one.

Lemma 3 Let $A$ and $B$ be metric spaces, $(T, \Sigma, \varphi)$ be a super-atomless probability space, $\tau$ be a tight Borel probability measure on $A \times B$ and $g: T \rightarrow A$ be a measurable function such that $\tau_{A}=\varphi \circ g^{-1}$. Then, there is a measurable function $f: T \rightarrow B$ such that $\tau=\varphi \circ(g, f)^{-1}$.

Proof. We claim that we may assume, without loss of generality, that $A$ and $B$ are compact metric spaces. In order to establish this claim, we first show if the conclusion of the lemma holds when $A$ and $B$ are Polish spaces, it also holds when they are arbitrary metric spaces.

In order to establish this latter claim, let $A$ and $B$ be arbitrary metric spaces. Since $\tau$ is tight, so are $\tau_{A}$ and $\tau_{B}$. Thus, we can find an increasing sequence $\left\{A_{n}\right\}_{n=1}^{\infty}$ of compact subsets of $A$ such that $\tau_{A}\left(\cup_{n} A_{n}\right)=1$, and an increasing sequence $\left\{B_{n}\right\}_{n=1}^{\infty}$ of compact subsets of $B$ such that $\tau_{B}\left(\cup_{n} B_{n}\right)=1$. Note that we must have $\tau\left(\left(\cup_{n} A_{n}\right) \times\right.$ $\left.\left(\cup_{n} B_{n}\right)\right)=1$ and $\left(\cup_{n} A_{n}\right) \times\left(\cup_{n} B_{n}\right)=\cup_{n}\left(A_{n} \times B_{n}\right)$, so we may view $\tau$ as a tight distribution on $\left(\cup_{n} A_{n}\right) \times\left(\cup_{n} B_{n}\right)$. Furthermore, changing $g$ on a null set if needed, we may assume that it takes all of its values in $\cup_{n} A_{n}$, and we may then assume as well that $A=\cup_{n} A_{n}$ and $B=\cup_{n} B_{n}$.

It then follows by Schwartz (1973, Corollary 2, p. 103, Corollary 3, p. 103, and Definition 2, p. 94) that there is a Polish topology $\eta_{A}$ on $A$ which is stronger than the original topology of $A$, but which generates the same Borel $\sigma$-algebra on $A$ as 
the original one. Similarly, there is a Polish topology $\eta_{B}$ on $B$, stronger than the original topology of $B$, but generating the same Borel $\sigma$-algebra on $B$ as the original topology of $B$. In particular, then, the product topology $\eta_{A} \times \eta_{B}$ is stronger than the original product topology of $A \times B$. Note also that since $\eta_{A}$ and $\eta_{B}$ are Polish topologies, the product of the Borel $\sigma$-algebras generated by $\eta_{A}$ and $\eta_{B}$ coincides with the Borel $\sigma$-algebra generated by the product topology $\eta_{A} \times \eta_{B}$. Consequently, the Borel $\sigma$-algebra generated by the product topology $\eta_{A} \times \eta_{B}$ coincides with the original Borel $\sigma$-algebra of $A \times B$. In view of these facts, we may assume that $A$ and $B$ are Polish spaces.

Finally, we show that if the conclusion of the lemma holds when $A$ and $B$ are compact metric spaces, it also holds when they are Polish spaces. Recall that if $C$ and $D$ are any two Polish spaces of the same cardinality, then they are Borel isomorphic, i.e., there is a bijection $\xi: C \rightarrow D$ such that both $\xi$ and its inverse $\xi^{-1}$ are Borel measurable, and recall that the cardinality of a Polish space is finite, countable infinite, or that of the continuum. Thus, since any compact metric space is a Polish space, we may assume, in fact, that both $A$ and $B$ are compact metric spaces. This establishes the above claim.

Let $x \mapsto \tau_{x}$ be a disintegration of $\tau$. Thus, for each $x \in A, \tau_{x}$ is a Borel probability measure on $B$, and for each Borel set $C \subseteq A \times B$,

$$
\tau(C)=\int_{A} \tau_{x}\left(C_{x}\right) \mathrm{d} \tau_{A}(x)
$$

where $C_{x} \subseteq B$ is the $x$-section of $C$. Note that for each $x \in A$ and each Borel set $C \subseteq A \times B$, writing $\delta_{x}$ for the Dirac measure at $x \in A$,

$$
\delta_{x} \otimes \tau_{x}(C)=\int_{A} \tau_{x^{\prime}}\left(C_{x^{\prime}}\right) \mathrm{d} \delta_{x}\left(x^{\prime}\right)=\tau_{x}\left(C_{x}\right)
$$

where the first equality follows by Fubini's theorem. Let $\phi: T \rightarrow \mathcal{M}(A \times B)$ be the mapping defined by $\phi(t)=\delta_{g(t)} \otimes \tau_{g(t)}$. Then $\phi$ is measurable in the sense that $t \mapsto \phi(t)(C)$ is measurable for each Borel set $C \subseteq A \times B$ (because $t \mapsto \phi(t)(C)$ is the composition of the measurable mapping $g$ with the measurable mapping $x \mapsto \tau_{x}\left(C_{x}\right)$ ), 
and for any Borel set $C \subseteq A \times B$,

$$
\begin{aligned}
& \int_{T} \phi(t)(C) \mathrm{d} \varphi(t)=\int_{T} \delta_{g(t)} \otimes \tau_{g(t)}(C) \mathrm{d} \varphi(t) \\
& =\int_{A} \delta_{x} \otimes \tau_{x}(C) \mathrm{d}\left(\varphi \circ g^{-1}\right)(x)=\int_{A} \delta_{x} \otimes \tau_{x}(C) \mathrm{d} \tau_{A}(x) \\
& =\int_{A} \tau_{x}\left(C_{x}\right) \mathrm{d} \tau_{A}(x)=\tau(C),
\end{aligned}
$$

where the two last equalities follow from (12) and (11), respectively. Since $(T, \Sigma, \varphi)$ is super-atomless, Corollary 1 in Podczeck (2007a) provides a measurable function $h: T \rightarrow A \times B$ such that $h(t) \in \operatorname{supp}(\phi(t))$ for almost all $t \in T$, and for all Borel sets $C \subseteq A \times B, \int_{T} \phi(t)(C) \mathrm{d} \varphi(t)=\varphi\left(h^{-1}(C)\right)$. Thus, it follows from above that $\tau=\varphi \circ h^{-1}$. For the function $h$ we can write $h=(e, f)$ with $e=\operatorname{proj}_{A} \circ h$ and $f=\operatorname{proj}_{B} \circ h$; in particular, both $e$ and $f$ are measurable and $\tau=\varphi \circ(e, f)^{-1}$. Also, for almost all $t \in T,(e(t), f(t))=h(t) \in \operatorname{supp}(\phi(t))=\operatorname{supp}\left(\delta_{g(t)} \otimes \tau_{g(t)}\right) \subseteq\{g(t)\} \times B$. Consequently $e(t)=g(t)$ for almost all $t \in T$, and hence $\tau=\varphi \circ(g, f)^{-1}$.

Lemma 3 immediately implies the following characterization of equilibrium distributions in games with super-atomless space of players.

Corollary 2 Let $(T, \Sigma, \varphi)$ be a super-atomless probability space. Then, $\tau$ is an equilibrium distribution of $G=((T, \Sigma, \varphi), V, X)$ if and only if there exists a Nash equilibrium $f$ of $G$ such that $\tau=\varphi \circ(V, f)^{-1}$.

\subsection{Proof of Theorem 1}

We start by establishing that condition 1 implies condition 2 . Let $K$ be an equicontinuous subset of $\mathcal{U}$ and $\varepsilon>0$. Let $\left\{x_{1}, \ldots, x_{m}\right\} \subseteq X$ be given by Lemma 2 and correspond to $K, M$ and $\varepsilon / 2$. Finally, let $N \in \mathbb{N}$ be such that $2 \omega_{K}\left(\frac{m^{2}+1}{n}\right)<\varepsilon / 2$ for all $n \geq N$. $^{7}$

Let $G_{n}$ be a game with a finite number of players with $V_{n}\left(T_{n}\right) \subseteq K$ and $n \geq N$. Consider the following game with a continuum of players: $G=(([0,1], \mathcal{B}([0,1]), \lambda), V, X)$ where $V(t)=V_{n}(i)$ if $t \in\left[\frac{i-1}{n}, \frac{i}{n}\right)$ for all $1 \leq i \leq n-1$ and $V(t)=V_{n}(n)$ if $t \in\left[\frac{n-1}{n}, 1\right]$.

\footnotetext{
${ }^{7}$ Recall that, for all $\delta>0, \omega_{K}(\delta)=\sup _{V \in K} \sup \{|V(x, \pi)-V(y, \tau)|: \max \{d(x, y), \rho(\pi, \tau)\} \leq \delta\}$.
} 
Note that $\lambda \circ V^{-1}=\nu_{n} \circ V_{n}^{-1} \in \mathcal{T}(\mathcal{U})$. By condition $1, G$ has an equilibrium distribution $\tau$ such that $\operatorname{supp}\left(\tau_{X}\right) \subseteq M$. Since $V([0,1])$ is a finite subset of $K$, it follows by Lemma 2 that $G$ has $\varepsilon / 2$ - equilibrium $f$ with $f([0,1]) \subseteq\left\{x_{1}, \ldots, x_{m}\right\}$.

By Lemma 1 , there exists a $\varepsilon / 2+2 \omega_{K}\left(\left(m^{2}+1\right) / n\right)$ - equilibrium $f_{n}$ of $G_{n}$. Since $2 \omega_{K}\left(\left(m^{2}+1\right) / n\right)<\varepsilon / 2$, then $f_{n}$ is an $\varepsilon$ - equilibrium of $G_{n}$. This concludes the proof that condition 1 implies condition 2.

The same scheme can be used to prove that condition 1 implies condition 3 . Let $\Gamma$ be a tight family of games with a finite set of players, $\varepsilon>0$ and $\eta>0$. Let $K$ be a compact subset of $\mathcal{U}$ satisfying $\nu_{n} \circ V_{n}^{-1}(K)>1-\eta$ for all $G_{n} \in \Gamma$. Then, let $\left\{x_{1}, \ldots, x_{m}\right\}$ be given by Lemma 2 and correspond to $K, M$ and $\varepsilon / 2$. Following the same arguments used above, we can show that $V_{n}(t)\left(f(t), \nu_{n} \circ f^{-1}\right) \geq$ $V_{n}(t)\left(x, \nu_{n} \circ\left(f \backslash{ }_{t} x\right)^{-1}\right)-\varepsilon$ for all $x \in X$ and all $t \in V_{n}^{-1}(K)$. Since, $\nu_{n} \circ V_{n}^{-1}(K)>1-\eta$ for all $G_{n} \in \Gamma$, the result follows.

We turn to the proof that condition 2 implies condition 1 . Let $\mu \in \mathcal{T}(\mathcal{U})$ be a game with a continuum of players. Since $\mu$ is tight, it has a separable support. Hence, it follows by Parthasarathy (1967, Theorem II.6.3, p.44) that there exists a sequence $\left\{\mu_{k}\right\}_{k} \in \mathcal{M}(\operatorname{supp}(\mu))$ converging to $\mu \in \mathcal{M}(\operatorname{supp}(\mu))$ and such that $\operatorname{supp}\left(\mu_{k}\right)$ is finite and $\mu_{k}(\{v\}) \in \mathbb{Q}$ for all $v \in \operatorname{supp}\left(\mu_{k}\right)$ and $k \in \mathbb{N}$. For all $k$, let $\operatorname{supp}\left(\mu_{k}\right)=$ $\left\{V_{k}^{1}, \ldots, V_{k}^{L_{k}}\right\} ;$ also, let $t_{k} \in \mathbb{N}$ and, for all $1 \leq l \leq L_{k}, \beta_{k}^{l} \in \mathbb{N}$ be such that $\beta_{k}^{l} / t_{k}=\mu_{k}\left(\left\{V_{k}^{l}\right\}\right)$.

Let $k \in \mathbb{N}$ be fixed. Then $\left\{V_{k}^{l}\right\}_{1 \leq l \leq L_{k}}$ is an equicontinuous subset of $\mathcal{U}$. Define, for all $\gamma \in \mathbb{N}$, a game $G_{\gamma t_{k}}=\left(\left(T_{\gamma t_{k}}, \nu_{\gamma t_{k}}\right), V_{\gamma t_{k}}, X\right)$ as follows: $G_{\gamma t_{k}}$ has $\gamma t_{k}$ players, each has $X$ as his choice set and their payoff functions are defined in the following way: $V_{\gamma t_{k}}: T_{\gamma t_{k}} \rightarrow \mathcal{U}$ is such that it associates $V_{k}^{l}$ to $\gamma \beta_{k}^{l}$ players, for all $1 \leq l \leq L_{k}$.

By condition 2, $G_{\gamma_{k} t_{k}}$ has a $1 / k$ - equilibrium $f_{\gamma_{k} t_{k}}: T_{\gamma_{k} t_{k}} \rightarrow M$ if we choose $\gamma_{k}$ sufficiently large. We may also choose $\gamma_{k}$ so that $\gamma_{k} t_{k}>k$, which implies that the sequence $\left\{\gamma_{k} t_{k}\right\}_{k=1}^{\infty}$ converges to infinity. Let $\tau_{k}=\nu_{\gamma_{k} t_{k}} \circ\left(V_{\gamma_{k} t_{k}}, f_{\gamma_{k} t_{k}}\right)^{-1} \in \mathcal{M}(\mathcal{U} \times X)$.

Since $\operatorname{supp}(\mu)$ is separable, $\left\{\tau_{\mathcal{U}, k}\right\}_{k}$ converges to $\mu$ and both $\mu$ and $\tau_{\mathcal{U}, k}$ are tight for all $k$, it follows that $\left\{\mu, \tau_{\mathcal{U}, 1}, \tau_{\mathcal{U}, 2}, \ldots\right\}$, and so $\left\{\tau_{\mathcal{U}, k}\right\}_{k}$ is tight by Hildenbrand (1974, Theorem 32, p. 49). Also, since $M$ is compact and $\operatorname{supp}\left(\tau_{k, X}\right) \subseteq M$ for all $k$, 
then $\left\{\tau_{X, 1}, \tau_{X, 2}, \ldots\right\}$ is tight. Thus, $\left\{\tau_{k}\right\}_{k}$ is tight (Hildenbrand (1974, Theorem 35, p. 50)) and, taking a subsequence if necessary, we may assume that $\left\{\tau_{k}\right\}$ converges (Hildenbrand (1974, Theorem 31, p. 49)). Let $\tau=\lim _{k} \tau_{k}$. Then, by Lemma 6, it follows that $\tau$ is an equilibrium distribution of $\tau_{\mathcal{U}}=\mu$. Furthermore, $\tau_{X}(M) \geq$ $\lim \sup _{k} \tau_{k, X}(M)=1$ and $\operatorname{sosupp}\left(\tau_{X}\right) \subseteq M$.

Similarly, we show that condition 3 implies condition 1 . We can use the same argument used in the proof that condition 2 implies condition 1, except that $f_{\gamma_{k} t_{k}}$ is only a $(1 / k, 1 / k)$ - equilibrium of $G_{\gamma_{k} t_{k}}$. However, Lemma 6 still applies and the conclusion follows.

\subsection{Proof of Theorem 2}

It follows from Corollary 2 that condition 1 implies condition 2. So, it suffices to show that condition 2 implies condition 1 . Let $\mu \in \mathcal{T}(\mathcal{U})$ be a game with a continuum of players. By Kallenberg (1997, Lemma 2.22, page 34), there exists a Borel measurable function $V:[0,1] \rightarrow \mathcal{U}$ such that $\mu=\lambda \circ V^{-1}$ (recall that $\lambda$ denotes the Lebesgue measure). By Podczeck (2007a, Appendix), there exists a super-atomless measure $\varphi$ on $[0,1]$ such that, denoting by $\Sigma$ the domain of $\varphi, \mathcal{B}([0,1]) \subseteq \Sigma$ and $\varphi$ agrees with $\lambda$ on $\mathcal{B}([0,1])$. Clearly, $V$ is still measurable when $\mathcal{B}([0,1])$ is replaced by $\Sigma$ and $\mu=\varphi \circ V^{-1}$. Indeed, for all measurable $C \subseteq \mathcal{U}, V^{-1}(C) \in \mathcal{B}([0,1])$, and so $\mu(C)=$ $\lambda\left(V^{-1}(C)\right)=\varphi\left(V^{-1}(C)\right)=\varphi \circ V^{-1}(C)$. By condition $2, G=(([0,1], \Sigma, \varphi), V, X)$ has a Nash equilibrium $f$. Hence, $\tau=\varphi \circ(V, f)^{-1}$ is an equilibrium distribution of $\mu$.

\subsection{Proof of Theorem 3}

Note that condition 2 trivially implies condition 3. Thus, it suffices to prove that condition 1 implies condition 2 and that condition 3 implies condition 1 .

Assume that condition 1 holds. Let $G=(([0,1], \mathcal{B}([0,1]), \lambda), V, X)$ be a game with a continuum of players with $V([0,1]) \subseteq \mathcal{U}$ and $C$ be a countable, closed subset of $X$ such that $M \cap C \neq \emptyset$. By condition 1, there exists an equilibrium distribution $\tau$ of $\mu=\lambda \circ V^{-1}$ relative to $C$. Note that $\operatorname{supp}\left(\tau_{X}\right) \subseteq C$, since $C$ is closed. It then follows 
by Khan and Sun (1995a, Theorem 2) that there exists a Nash equilibrium $f$ of $G$ relative to $C$ such that $\tau=\lambda \circ(V, f)^{-1}$. Thus, condition 2 holds.

Assume that condition 3 holds. Let $\mu \in \mathcal{T}(\mathcal{U})$ be a game with a continuum of players and let $S$ be a closed subset of $X$ such that $M \cap S \neq \emptyset$. Let $C=\left\{x_{m}\right\}_{m=1}^{\infty}$ be a countable dense subset of $S$. We claim that it suffices to establish that there exists an equilibrium distribution $\tau$ of $\mu$ relative to $C$. In fact, given such a distribution $\tau$, since $C$ is dense in $S$, it follows that $\operatorname{supp}\left(\tau_{X}\right) \subseteq \bar{C}=S$ and that the set $\{(u, x) \in$ $\left.\mathcal{U} \times X: u\left(x, \tau_{X}\right) \geq u\left(S, \tau_{X}\right)\right\}$ is equal to $\left\{(u, x) \in \mathcal{U} \times X: u\left(x, \tau_{X}\right) \geq u\left(C, \tau_{X}\right)\right\}$. Hence, $\tau\left(\left\{(u, x) \in \mathcal{U} \times X: u\left(x, \tau_{X}\right) \geq u\left(S, \tau_{X}\right)\right\}=1\right.$ and so $\tau$ is an equilibrium distribution of $\mu$ relative to $S$.

We then establish the existence of an equilibrium distribution $\tau$ of $\mu$ relative to $C$. Since $\operatorname{supp}(\mu)$ is a closed (hence, complete) and separable subset of $\mathcal{U}$, it follows by Kallenberg (1997, Lemma 2.22, page 34) that there exists a Borel measurable function $V:[0,1] \rightarrow \mathcal{U}$ such that $\mu=\lambda \circ V^{-1}$ and so we can represent the game $\mu$ by $G=(([0,1], \mathcal{B}([0,1]), \lambda), V, X)$. For all $k \in \mathbb{N}$, define $F_{k}=\left\{x_{1}, \ldots, x_{k}\right\}$. By condition 3 , there exists a Nash equilibrium $f_{k}$ of $G$ relative to $F_{k}$ for all $k$. Let $\tau_{k}=\lambda \circ\left(V, f_{k}\right)^{-1}$ for all $k$. Since $\tau_{k, \mathcal{U}}=\lambda \circ V^{-1}$ and $\operatorname{supp}\left(\tau_{k, X}\right) \subseteq \bar{C} \subseteq M$ for all $k$, it follows that the sequence $\left\{\tau_{k}\right\}_{k}$ is tight and so, taking a subsequence if necessary, we may assume that it converges. Let $\tau=\lim _{k} \tau_{k}$. Clearly, $\operatorname{supp}\left(\tau_{X}\right) \subseteq \bar{C} \subseteq M$. We next establish that $\tau$ is an equilibrium distribution of $\mu$ relative to $C$.

Let $(u, x) \in \operatorname{supp}(\tau)$ and $m \in \mathbb{N}$. For all $k \in \mathbb{N}$, define $A_{k}=\{(u, x) \in \mathcal{U} \times X$ : $u\left(x, \tau_{k, X}\right)<u\left(y, \tau_{k, X}\right)$ for some $\left.y \in F_{k}\right\}$. Since $\tau_{k}$ is an equilibrium distribution of $G$ relative to $F_{k}$, it follows that $\tau_{k}\left(A_{k}\right)=0$ for all $k \in \mathbb{N}$. By Lemma 7 , there exists a subsequence $\left\{\tau_{k_{j}}\right\}_{j=1}^{\infty}$ of $\left\{\tau_{k}\right\}_{k=1}^{\infty}$ and, for each $j \in \mathbb{N},\left(u_{j}, x_{j}\right) \in \operatorname{supp}\left(\tau_{k_{j}}\right) \backslash A_{k_{j}}$ such that $\lim _{j}\left(u_{j}, x_{j}\right)=(u, x)$. Then, $u_{j}\left(x_{j}, \tau_{k_{j}, X}\right) \geq u_{j}\left(x_{m}, \tau_{k_{j}, X}\right)$ for all $j \in \mathbb{N}$ such that $k_{j} \geq m$. Since $\lim _{j} u_{j}=u, \lim _{j} \tau_{k_{j}}=\tau$, and $\lim _{j} x_{j}=x$, it follows that $u\left(x, \tau_{X}\right) \geq u\left(x_{m}, \tau_{X}\right)$. Since $(u, x) \in \operatorname{supp}(\tau)$ and $m \in \mathbb{N}$ were chosen arbitrarily, it follows that $\operatorname{supp}(\tau) \subseteq\left\{(u, x) \in \mathcal{U} \times X: u\left(x, \tau_{X}\right) \geq u\left(x_{m}, \tau_{X}\right)\right.$ for all $\left.m \in \mathbb{N}\right\}$ and so $\tau$ is an equilibrium distribution of $\mu$ relative to $C$. 


\subsection{On the Asymptotic Existence Result}

In this section, we show that in general, we cannot obtain a pure Nash equilibrium even in equicontinuous large games. The exact version of condition 1 in Corollary 1 (i.e., with $\varepsilon=0$ ) therefore holds only in the limit case of games with a continuum of players. As an easy consequence, we also show that it is not possible to dispense with the equicontinuity assumption used in that condition.

In order to establish the above claim, we show that there exists an equicontinuous subset $K$ of $\mathcal{U}$ and $m \in \mathbb{N}$ such that for all $N \in \mathbb{N}$ there exists a game $G_{n}=$ $\left(\left(T_{n}, \Sigma_{n}, \nu_{n}\right), V_{n}, X\right)$ with $n \geq N$ and $V_{n}\left(T_{n}\right) \subseteq K$ which has no Nash equilibrium.

Let $X=\{(1,0),(0,1)\}=\left\{e_{1}, e_{2}\right\} \subseteq \mathbb{R}^{2}$, and for all $N \in \mathbb{N}$, we let $n \geq N$ be a multiple of 4 (e.g., $n=4 N)$. Define $G_{n}$ as follows: let $T_{1}=\{1, \ldots, n / 2\}$ and $T_{2}=\{n / 2+1, \ldots, n\}$. For $t \in T_{1}$, let

$$
\begin{aligned}
& V(t)\left(e_{1}, \mu\right)=\frac{1}{2}-\frac{n \mu_{1}-1}{n-1} \text { and } \\
& V(t)\left(e_{2}, \mu\right)=\frac{n \mu_{1}}{n-1}-\frac{1}{2},
\end{aligned}
$$

while for $t \in T_{2}$, let $V(t)\left(e_{i}, \mu\right)=-V(1)\left(e_{i}, \mu\right), i=1,2$. Note that for all $t \in T_{1}$ and strategy $f=\left(f_{1}, f_{2}\right)$,

$$
\begin{aligned}
& U(t)\left(f \backslash_{t} e_{1}\right)=\frac{1}{2}-\sum_{j \neq t} \frac{f_{1}(j)}{n-1} \text { and } \\
& U(t)\left(f \backslash_{t} e_{2}\right)=\sum_{j \neq t} \frac{f_{1}(j)}{n-1}-\frac{1}{2} .
\end{aligned}
$$

Thus, for $t \in T_{1}$, we have that player $t$ 's optimal choice is:

$$
f_{1}(t)= \begin{cases}1 & \text { if } \sum_{j \neq t} \frac{f_{1}(j)}{n-1}<\frac{1}{2} \\ x \in\{0,1\} & \text { if } \sum_{j \neq t} \frac{f_{1}(j)}{n-1}=\frac{1}{2} \\ 0 & \text { if } \sum_{j \neq t} \frac{f_{1}(j)}{n-1}>\frac{1}{2}\end{cases}
$$

For any player $t \in T_{2}$, the optimal choice is just the opposite, that is, player $t$ 's optimal choice is:

$$
f_{1}(t)= \begin{cases}0 & \text { if } \sum_{j \neq t} \frac{f_{1}(j)}{n-1}<\frac{1}{2} \\ x \in\{0,1\} & \text { if } \sum_{j \neq t} \frac{f_{1}(j)}{n-1}=\frac{1}{2} \\ 1 & \text { if } \sum_{j \neq t} \frac{f_{1}(j)}{n-1}>\frac{1}{2}\end{cases}
$$


Let $K=\{V(1),-V(1)\}$.

We claim that $G_{n}$ has no pure strategy Nash equilibrium. Let

$$
\bar{x}=\sum_{t \in T} \frac{f_{1}(t)}{n},
$$

and

$$
\bar{x}_{t}=\sum_{j \neq t} \frac{f_{1}(j)}{n-1}=\frac{n \bar{x}-f_{1}(t)}{n-1},
$$

for any $t \in T$. We consider the cases $\bar{x}<1 / 2, \bar{x}=1 / 2$ and $\bar{x}>1 / 2$.

Assume that $f$ is pure and that $\bar{x}=1 / 2$. If $t \in T_{2}$ is such that $f_{1}(t)=0$, then $\bar{x}_{t}=n /(2 n-2)>1 / 2$. Therefore, the gain from deviating to $e_{1}$ is

$$
2 \bar{x}_{t}-1=\frac{1}{n-1}>0 .
$$

Similarly, if $t \in T_{2}$ is such that $f_{1}(t)=1$, then $\bar{x}_{t}=(n-2) /(2 n-2)<1 / 2$, and player $t$ gains by deviating to $e_{2}$.

If $\bar{x}<1 / 2$, then $\bar{x} \leq 1 / 2-1 / n$ and there exists $t \in T_{1}$ such that $f_{1}(t)=0$. It follows that $\bar{x}_{t}<1 / 2$, implying that player $t$ is not optimizing. Finally, if $\bar{x}>1 / 2$, then $\bar{x} \geq 1 / 2+1 / n$ and there exists $t \in T_{1}$ such that $f_{1}(t)=1$. Thus, $\bar{x}_{t}>1 / 2$ and so player $t$ is not optimizing. It follows from the analysis of the above three cases that $G_{n}$ has no pure strategy Nash equilibrium.

The above example can be modified to show that we cannot dispense with the equicontinuity assumption in condition 1 in Corollary 1.

Let $n \in \mathbb{N}$ and $G_{n}$ be as defined above. Since $G_{n}$ has no pure strategy Nash equilibria, it follows that there exists $\varepsilon_{n}>0$ with the property that no pure strategy is an $\varepsilon_{n}$ - equilibrium of $G_{n}$. In fact, the set of pure strategy is compact (it can be identified with $\left.\left\{e_{1}, e_{2}\right\}^{n}\right)$; thus, if this claim were to be false, we would obtain a pure strategy Nash equilibrium from a sequence of pure $\varepsilon_{n}$ - equilibria with the corresponding sequence of $\varepsilon_{n}$ converging to zero. Thus, define $\tilde{G}_{n}=\left(\left(T_{n}, \Sigma_{n}, \nu_{n}\right), \tilde{V}_{n}, X\right)$ by letting $\tilde{V}(t)=V(t) / \varepsilon_{n}$ for all $t \in T_{n}$. Then, it follows that $\tilde{G}_{n}$ has no pure strategy 1 - equilibria.

Note that it follows by Corollary 1 that $\varepsilon_{n} \rightarrow 0$. This shows that the family of payoff functions we used in the example is not equicontinuous and accounts for the 
failure of the conclusion of Theorem 1 .

Finally, we note that the games used in the above examples are such that each player's payoff depends only on his choice and the average choice of the others. This shows that even if we restrict attention to this class of games, we cannot obtain a pure $\varepsilon$ - equilibrium for all $\varepsilon>0$ for all sufficiently large games without equicontinuity. This last conclusion generalizes the result in Carmona (2004b): since for the nonequicontinuous game in our example there is no pure $\varepsilon$ - equilibrium for all $\varepsilon>0$ small enough, then it follows that no mixed strategy equilibrium can be $\varepsilon$ - purified.

\subsection{On the Necessity of Compact Spaces}

In this subsection, we show that the action space being compact is not only sufficient but also necessary for the existence of equilibria when $\mathcal{U}=\mathcal{C}$ and $X$ is separable and complete.

Theorem 4 Suppose that $X$ is a complete, separable metric space. The following conditions are equivalent:

1. $X$ is compact.

2. For all games with a continuum of players $\mu \in \mathcal{T}(\mathcal{C})$, there exists an equilibrium distribution $\tau$ of $\mu$.

3. For all equicontinuous subsets $K$ of $\mathcal{C}$ and $\varepsilon>0$, there exists $m, N \in \mathbb{N}$ and $\left\{x_{1}, \ldots, x_{m}\right\} \subseteq M$ such that for all $n \geq N$, all games with a finite number of players $G_{n}=\left(\left(T_{n}, \Sigma_{n}, \nu_{n}\right), V_{n}, X\right)$ with $V_{n}\left(T_{n}\right) \subseteq K$ have an $\varepsilon$ - equilibrium $f_{n}$ satisfying $f_{n}\left(T_{n}\right) \subseteq\left\{x_{1}, \ldots, x_{m}\right\}$.

Proof. We start by showing that condition 1 is equivalent to condition 2 . If $X$ is compact, i.e., condition 1 holds, it follows from Mas-Colell (1984, Theorem 1) that condition 2 holds. Conversely, suppose that condition 1 does not hold. Then, there exists a continuous, bounded function $f: X \rightarrow \mathbb{R}_{+}$that does not attain its maximum in $X$. In fact, there exists an unbounded, continuous function $h: X \rightarrow \mathbb{R}_{+}$(see Lima 
(1993, Example 16, p. 224)). Then, let $\bar{d}$ be a metric in $\overline{\mathbb{R}}_{+}$and define $g: \overline{\mathbb{R}}_{+} \rightarrow \mathbb{R}$ by $g(x)=-\bar{d}(x, \infty) /(1+\bar{d}(x, \infty))$ and $f: X \rightarrow \mathbb{R}$ by $f=g \circ h$. Clearly, $f(X) \subseteq[-1,0]$.

Note that $g$ is continuous and so is $h$ when viewed as a function to $\overline{\mathbb{R}}_{+}$. This implies that $f$ is also continuous. Since $h$ is unbounded, then $\sup _{x \in X} f(x)=0$, but $f(x)<0$ since $h(X) \subseteq \mathbb{R}$. Hence, $f$ is a bounded, continuous, real-valued function that does not attain its maximum in $X$.

Thus, let $\tilde{v} \in \mathcal{C}$ be defined by $\tilde{v}(x, \phi)=f(x)$ for all $x \in X$ and $\phi \in \mathcal{M}(X)$ and $\mu \in \mathcal{T}(\mathcal{C})$ by $\mu(\{\tilde{v}\})=1$. Then, $\mu$ has no equilibrium distribution. In conclusion, condition 2 does not hold.

Suppose next that condition 2 holds. Then, by the above, $X$ is compact and it follows that condition 2 in Theorem 1 holds when $\mathcal{U}=\mathcal{C}$ and $M=X$. Then, condition 3 follows from Theorem 1.

Finally, we show that condition 3 implies condition 1 . Assume that $X$ is not compact. We may assume the metric $d$ on $X$ is bounded (if not, replace it by the equivalent metric $\bar{d}$ defined by $\bar{d}(x, y)=d(x, y) /(1+d(x, y))$ for all $x, y \in X)$. Since $X$ is complete, then $X$ is not totally bounded. For all $x \in X$, let $v_{x} \in \mathcal{C}$ be defined by $v_{x}\left(x^{\prime}, \phi\right)=-d\left(x^{\prime}, x\right)$ for all $x^{\prime} \in X$ and $\phi \in \mathcal{M}(X)$ and define $K=\left\{v_{x}\right\}_{x \in X}$. Clearly, $K$ is equicontinuous.

Since $X$ is not totally bounded, there exists $\varepsilon>0$ such that for all finite subsets $F$ of $X$, there exists $x \in X$ such that $d\left(x, x^{\prime}\right)>\varepsilon$ for all $x^{\prime} \in F$. Let $m, n \in \mathbb{N}$ and $\left\{x_{1}, \ldots, x_{m}\right\} \subseteq X$ be given and let $x \in X$ be such that $d\left(x, x^{\prime}\right)>\varepsilon$ for all $x^{\prime} \in\left\{x_{1}, \ldots, x_{m}\right\}$. Then, letting $n=N$ and $G_{n}$ be such that $v_{n}(t)=v_{x}$ for all $t \in T_{n}$, it follows that if $f_{n}$ is an $\varepsilon$ - equilibrium of $G_{n}$, then $f_{n}(t) \notin\left\{x_{1}, \ldots, x_{m}\right\}$. In conclusion, condition 2 does not hold.

\section{Concluding Remarks}

In this paper, we have considered several existence results for large games with the purpose of establishing their equivalence. In our view, such equivalence is important since it expresses the close relationship between the different formalizations of large 
games and their corresponding equilibrium notions. In particular, all the existence results are equally strong and so none of the formalizations we consider should be regarded as better suited to address the existence problem of large games.

Furthermore, our equivalence results also imply that the relative strengths and weaknesses of the different equilibrium concepts and formalizations are more apparent than substantial. In fact, as their proofs make clear, it is possible to obtain an equilibrium in one model using an equilibrium (or a sequence of equilibria) in another one. Thus, a critique (resp. praise) to a particular equilibrium concept in some given formalization, implies a critique (resp. praise) to all the other equilibrium concepts (from which the original one can be obtained).

To illustrate this point consider a game with an uncountable action space. Then, an equilibrium strategy will exist if the space of players is super-atomless. However, an equilibrium distribution exists under exactly the same assumptions. Furthermore, all games with the same distribution of players' characteristics as the original game but with a Lebesgue space of players have an equilibrium relative to a (finitely) discretisized action space, and, by choosing the discrete action space close to the original one, these strategies can be made arbitrarily close to an equilibrium distribution of the original game. Thus, as long as one is only interested in the strategic behavior displayed in such equilibrium, all three methods of reaching such equilibrium should deserve the same appraisal.

In conclusion, as far as the existence problem is concerned, all the formalizations of large games that we have considered should be regarded as equivalent and the choice of which one to use in practice regarded as a matter of taste and convenience.

\section{A Appendix}

In this appendix, we prove several results needed for our main results. Lemma 4 deals with measures with a finite support, which can be thought of as a vector in some Euclidean space. Roughly, Lemma 4 says that the Prohorov distance between two measures whose support is contained in some finite set is proportional to their 
Euclidean distance.

Lemma 4 Let $\tau, \mu \in \mathcal{M}(X)$ be such that $\operatorname{supp}(\tau) \cup \operatorname{supp}(\mu) \subseteq \Psi$, where $\Psi$ is a finite set. If there exists $\varepsilon>0$ such that $\left|\tau_{l}-\mu_{l}\right| \leq \varepsilon$ for all $1 \leq l \leq|\Psi|$, then $\rho(\tau, \mu) \leq|\Psi| \varepsilon$.

Proof. Let $\varepsilon>0$ and $B \subseteq X$ be Borel measurable. Then,

$$
\tau(B)=\sum_{l \in \Psi \cap B} \tau_{l} \leq \sum_{l \in \Psi \cap B}\left(\mu_{l}+\varepsilon\right) \leq \sum_{l \in \Psi \cap B} \mu_{l}+|\Psi| \varepsilon \leq \mu\left(\bar{B}_{|\Psi| \varepsilon}(B)\right)+|\Psi| \varepsilon .
$$

Similarly, we can show that $\mu(B) \leq \tau\left(\bar{B}_{|\Psi| \varepsilon}(B)\right)+|\Psi| \varepsilon$. This implies that $\rho(\tau, \mu) \leq$ $|\Psi| \varepsilon$.

Lemma 5 shows that in large games, deviations by a small fraction of players have a small impact on the distribution of actions.

Lemma 5 Let $G_{n}$ be a game with a finite number of players and let $f$ and $g$ be strategies. If $\left|\left\{t \in T_{n}: f(t) \neq g(t)\right\}\right| / n \leq \gamma$, then $\rho\left(\nu_{n} \circ f^{-1}, \nu_{n} \circ g^{-1}\right) \leq \gamma$.

Proof. Let $\mu=\nu_{n} \circ f^{-1}$ and $\tau=\nu_{n} \circ g^{-1}$. Let $B \subseteq X$ be Borel measurable. Then,

$$
\begin{aligned}
\tau(B) & =\frac{|\{t: g(t) \in B\}|}{n} \leq \frac{|\{t: f(t) \in B\}|}{n}+\frac{|\{t: f(t) \neq g(t)\}|}{n} \\
& =\mu(B)+\frac{|\{t: f(t) \neq g(t)\}|}{n} \leq \mu\left(\bar{B}_{\gamma}(B)\right)+\gamma .
\end{aligned}
$$

Similarly, we can show that $\mu(B) \leq \tau\left(\bar{B}_{\gamma}(B)\right)+\gamma$. This implies that $\rho(\tau, \mu) \leq \gamma$.

In particular, we have that $\rho\left(\nu_{n} \circ f^{-1}, \nu_{n} \circ\left(f \backslash_{t} x\right)^{-1}\right) \leq 1 / n$ for all strategies $f$, players $t \in T_{n}$ and actions $x \in X$.

Lemma 6 draws conclusions for games with a continuum of players from properties of large finite games and was used in Lemma 1. It considers a more general case in which a game $G_{n}=\left(\left(T_{n}, \Sigma_{n}, \nu_{n}\right), V_{n}, X\right)$ with finitely many players has $\left|T_{n}\right|$ players (not necessarily equal to $n$ ), and $\nu_{n}$ is the uniform measure on $T_{n}$.

Lemma 6 Let $G=((T, \Sigma, \varphi), V, X)$ be a game with a continuum of players, $\tau$ be a distribution on $\mathcal{C} \times X$ satisfying $\tau_{\mathcal{C}}=\lambda \circ V^{-1} \in \mathcal{T}(\mathcal{C})$ and $\varepsilon \geq 0$. Suppose that $\left\{G_{n}\right\}_{n=1}^{\infty}$ is a sequence of games with a finite number of players and $\left\{f_{n}\right\}_{n=1}^{\infty}$ is a 
sequence of strategies such that $\left|T_{n}\right| \rightarrow \infty, f_{n}$ is an $\left(\varepsilon_{n}, \eta_{n}\right)$ - equilibrium of $G_{n}$ for all $n, \lim _{n} \varepsilon_{n}=\varepsilon, \lim _{n} \eta_{n}=0$ and $\lim _{n} \nu_{n} \circ\left(V_{n}, f_{n}\right)^{-1}=\tau$, then $\tau$ is an $\varepsilon$ - equilibrium distribution of $G$.

This lemma can be established using an argument analogous to the one employed to prove that condition 3 implies 2 in Theorem 3. Both results rely on the following lemma.

Lemma 7 Let $Z$ be a metric space, $\left\{\tau_{k}\right\}_{k=1}^{\infty}$ be a sequence in $\mathcal{T}(Z)$ converging to $\tau \in \mathcal{T}(Z)$, and $\left\{A_{k}\right\}_{k=1}^{\infty}$ be a sequence of Borel subsets of $Z$ with $\lim _{k} \tau_{k}\left(A_{k}\right)=0$. Then, for all $z \in \operatorname{supp}(\tau)$, there exists a subsequence $\left\{\tau_{k_{j}}\right\}_{j=1}^{\infty}$ of $\left\{\tau_{k}\right\}_{k=1}^{\infty}$ and an element $z_{j} \in \operatorname{supp}\left(\tau_{k_{j}}\right) \backslash A_{k_{j}}$ for all $j \in \mathbb{N}$ such that $\lim _{j} z_{j}=z$.

Proof. Let $z \in \operatorname{supp}(\tau)$ and suppose the assertion were false. Then there is an open neighborhood $U$ of $z$ such that $U \cap\left(\operatorname{supp}\left(\tau_{k}\right) \backslash A_{k}\right)=\emptyset$ for all sufficiently large $k$. Thus, $U \cap \operatorname{supp}\left(\tau_{k}\right) \subseteq A_{k}$ for all sufficiently large $k$ and hence $\lim _{k} \tau_{k}\left(U \cap \operatorname{supp}\left(\tau_{k}\right)\right)=0$. Since $\tau_{k}(U)=\tau_{k}\left(U \cap \operatorname{supp}\left(\tau_{k}\right)\right)+\tau_{k}\left(U \backslash \operatorname{supp}\left(\tau_{k}\right)\right)=\tau_{k}\left(U \cap \operatorname{supp}\left(\tau_{k}\right)\right)$, it follows that $0 \leq \tau(U) \leq \liminf _{k} \tau_{k}(U)=\lim _{k} \tau_{k}(U)=0$. Hence, $\tau(U)=0$, contradicting the hypothesis that $z \in \operatorname{supp}(\tau)$.

\section{References}

Al-NajJAR, N. (2007): "Large Games and the Law of Large Numbers," forthcoming, Games and Economic Behavior.

BAlder, E. (2002): "A Unifying Pair of Cournot-Nash Equilibrium Existence Results," Journal of Economic Theory, 102, 437-470.

Carmona, G. (2004a): "Nash Equilibria of Games with a Continuum of Players," Universidade Nova de Lisboa.

(2004b): "On the Purification of Nash Equilibria of Large Games," Economics Letters, 85, 215-219. 
(2008): "Large Games with Countable Characteristics," Journal of Mathematical Economics, 44, 344-347.

Fremlin, D. (2003): Measure Theory, vol. 4: Topological Measure Spaces. Torres Fremlin, Colchester.

Hildenbrand, W. (1974): Core and Equilibria of a Large Economy. Princeton University Press, Princeton.

Kalai, E. (2004): "Large Robust Games," Econometrica, 72, 1631-1665.

Kallenberg, O. (1997): Foundations of Modern Probability. Springer, Berlin.

Khan, M., K. Rath, and Y. Sun (1997): "On the Existence of Pure Strategy Equilibria in Games with a Continuum of Players," Journal of Economic Theory, $76,13-46$.

Khan, M., And Y. Sun (1995a): "The Marriage Lemma and Large Anonymous Games with Countable Actions," Mathematical Proceedings of the Cambridge Philosophical Society, 117, 385-387.

- (1995b): "Pure Strategies in Games with Private Information," Journal of Mathematical Economics, 24, 633-653.

_ (1999): "Non-Cooperative Games on Hyperfinite Loeb Spaces," Journal of Mathematical Economics, 31, 455-492.

Lima, E. L. (1993): Espaços Métricos. IMPA, Rio de Janeiro, 3rd edn.

Mas-Colell, A. (1984): "On a Theorem by Schmeidler," Journal of Mathematical Economics, 13, 201-206.

Nash, J. (1950): "Non-Cooperative Games," Ph.D. thesis, Princeton University.

Parthasarathy, K. (1967): Probability Measures on Metric Spaces. Academic Press, New York. 
Podczeck, K. (2007a): "On Purification of Measure-valued Maps," forthcoming, Economic Theory.

(2007b): "On the Convexity and Compactness of the Integral of a Banach Space Valued Correspondence," forthcoming, Journal of Mathematical Economics.

RAshid, S. (1983): "Equilibrium Points of Non-atomic Games: Asymptotic Results," Economics Letters, 12, 7-10.

Rudin, W. (1976): Principles of Mathematical Analysis. McGraw-Hill, New York.

SchmeIdleR, D. (1973): "Equilibrium Points of Nonatomic Games," Journal of Statistical Physics, 4, 295-300.

Schwartz, L. (1973): Radon Measures on Arbitrary Topological Spaces and Cylindrical Measures. Oxford University Press, Oxford.

Wooders, M., E. Cartwright, and R. Selten (2006): "Behavioral Conformity in Games with Many Players," Games and Economic Behavior, 57, 347-360. 